\title{
Advances in liquid phase soft-x-ray photoemission spectroscopy: A new experimental setup at BESSY II
}

Robert Seidel, Marvin N. Pohl, Hebatallah Ali, Bernd Winter, and Emad F. Aziz

Citation: Review of Scientific Instruments 88, 073107 (2017); doi: 10.1063/1.4990797

View online: http://dx.doi.org/10.1063/1.4990797

View Table of Contents: http://aip.scitation.org/toc/rsi/88/7

Published by the American Institute of Physics

\section{Articles you may be interested in}

Manipulating motions of targeted single cells in solution by an integrated double-ring magnetic tweezers imaging microscope

Review of Scientific Instruments 88, 073703 (2017); 10.1063/1.4995362

Solid-state repetitive generator with a gyromagnetic nonlinear transmission line operating as a peak power amplifier

Review of Scientific Instruments 88, 074703 (2017); 10.1063/1.4993732

Stabilizing low-frequency oscillation with two-stage filter in Hall thrusters Review of Scientific Instruments 88, 073502 (2017); 10.1063/1.4990045

NanoRocks: Design and performance of an experiment studying planet formation on the International Space Station

Review of Scientific Instruments 88, 074502 (2017); 10.1063/1.4991857

Measurement of the velocity of neutral fragments by the "correlated ion and neutral time of flight" method combined with "velocity-map imaging"

Review of Scientific Instruments 88, 083101 (2017); 10.1063/1.4991828

TinyLev: A multi-emitter single-axis acoustic levitator

Review of Scientific Instruments 88, 085105 (2017); 10.1063/1.4989995

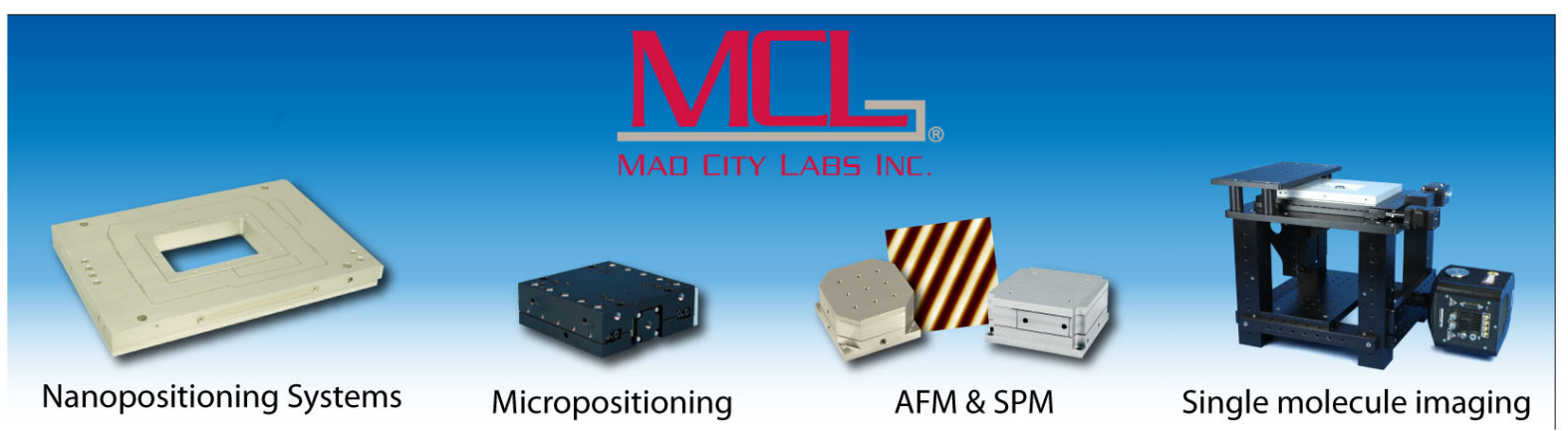




\title{
Advances in liquid phase soft-x-ray photoemission spectroscopy: A new experimental setup at BESSY II
}

\author{
Robert Seidel, ${ }^{1, a)}$ Marvin N. Pohl, ${ }^{1,2, b)}$ Hebatallah Ali, ${ }^{1,2}$ Bernd Winter, ${ }^{1, b)}$ \\ and Emad F. Aziz ${ }^{1,2,3, a)}$ \\ ${ }^{1}$ Helmholtz-Zentrum Berlin für Materialien und Energie, Methods for Material Development, \\ Albert-Einstein-Straße 15, D-12489 Berlin, Germany \\ ${ }^{2}$ Department of Physics, Freie Universität Berlin, Arnimallee 14, D-14159 Berlin, Germany \\ ${ }^{3}$ School of Chemistry, Monash University, Clayton 3800, Victoria, Australia
}

(Received 11 November 2016; accepted 17 June 2017; published online 31 July 2017)

A state-of-the-art experimental setup for soft X-ray photo- and Auger-electron spectroscopy from liquid phase has been built for operation at the synchrotron-light facility BESSY II, Berlin. The experimental station is named $\mathrm{SOL}^{3}$, which is derived from solid, solution, and solar, and refers to the aim of studying solid-liquid interfaces, optionally irradiated by photons in the solar spectrum. $\mathrm{SOL}^{3}$ is equipped with a high-transmission hemispherical electron analyzer for detecting electrons emitted from small molecular aggregates, nanoparticles, or biochemical molecules and their components in (aqueous) solutions, either in vacuum or in an ambient pressure environment. In addition to conventional energy-resolved electron detection, $\mathrm{SOL}^{3}$ enables detection of electron angular distributions by the combination of $\mathrm{a} \pm 11^{\circ}$ acceptance angle of the electron analyzer and a rotation of the analyzer in the polarization plane of the incoming synchrotron-light beam. The present manuscript describes the technical features of $\mathrm{SOL}^{3}$, and we also report the very first measurements of soft-X-ray photoemission spectra from a liquid microjet of neat liquid water and of $\mathrm{TiO}_{2}$-nanoparticle aqueous solution obtained with this new setup, highlighting the necessity for state-of-the-art electron detection. Published by AIP Publishing. [http://dx.doi.org/10.1063/1.4990797]

\section{INTRODUCTION}

Within only one decade, photoelectron (PE) spectroscopy (also referred to as photoemission) from aqueous solutions has developed into a vivid field of research. ${ }^{1-10}$ The introduction of liquid microjets has enabled the application of PE spectroscopy to highly volatile liquid-phase samples under vacuum conditions. A sketch of the experiment is shown in Fig. 1(A). Liquid-jet valence PE spectra have mostly been measured from aqueous solutions, ${ }^{4}$ revealing the lowest ionization and electron detachment energies which are key quantities for understanding aqueous-phase chemical reactions, and moreover, the lowest-energy PE peak from a solution can be related to its reorganization energy connected with the change of the solute charge state. ${ }^{11,12}$ Liquid-jet core-level PE spectroscopy has been used to probe with large sensitivity the local chemical environment of solutes, which depends, for example, on solute concentration, counter ions, ionic strength, $\mathrm{pH}$, and density depth-profile (typically across the solutionvacuum interface). ${ }^{13-16}$ Local structure variations reflect in changes of the respective core-level binding energies, the chemical shifts. ${ }^{15,17}$ A particularly vital application of corelevel PE spectroscopy is the so-called depth-profile measurement. ${ }^{16,18,19}$ Here one exploits the electron-energy dependent travel length, the electron elastic and inelastic mean free paths

\footnotetext{
a) Authors to whom correspondence should be addressed: robert.seidel@ helmholtz-berlin.de and emad.aziz@ helmholtz-berlin.de

b) Current address: Fritz-Haber-Institut der Max-Planck-Gesellschaft, Faradayweg 4-6, D-14195 Berlin, Germany.
}

in aqueous solutions, to tune the sensitivity of this method from an essentially surface-sensitive to a rather bulk-solution sensitive measurement. Less explored, but of large importance for understanding the effect of hydrogen bonding on solute electronic structure, and also for the characterization of molecular orientation at the solution interface, are photoelectron angular distributions (PADs) ${ }^{20}$ which will be described below in more detail.

Liquid-jet photoemission also comprises the detection of electrons emitted due to second-order processes which are the electronic relaxations, resulting, for instance, in the emission of Auger electrons. ${ }^{2}$ These autoionization processes (of which Auger-electron decay is only the local case) were found to be of enormous importance for liquid water and hydrogenbonded systems in general. ${ }^{21,22}$ Auger spectra, measured at a given core-level resonance, play a key role in identifying the bonding interactions in the case of strong hybridization, as in transition-metal (TM)-water complexes. Specifically, the electronic relaxations following metal $2 \mathrm{p}-3 \mathrm{~d} \mathrm{X}$-ray absorption, metal $2 p-3 d$ transitions, give rise to strong signal enhancements of peaks in the valence PE spectrum from which solute and solvent orbital mixing can be identified, entirely based on experimental grounds. ${ }^{23,24}$ The enhancement results from the interference of electrons with the same kinetic energy, but originating from different processes, the (direct) photoionization and the Auger decay. The final (autoionization) states are indistinguishable. In the case of weak hydrogen-bonding interactions, also non-local autoionization processes occur, revealing information on hydrogen-bond geometry and strength as well as on ultrafast nuclear dynamics. One process that has gained 


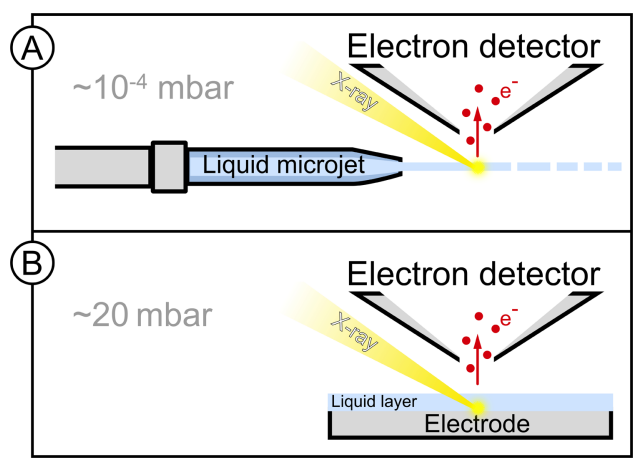

FIG. 1. Different methods to investigate liquid samples in the $\mathrm{SOL}^{3}$ interaction chamber. (A) Liquid-microjet technique using a capillary for creating a laminar flow which is irradiated by soft X-rays. (B) Liquid layer condensed on an electrode surface; the film is equilibrated under ambient-pressure conditions. Soft X-rays are used for the photoemission experiments from the liquid-solid interface.

particular attention is the (core-level) ionization-induced Intermolecular Coulombic Decay (ICD), a non-local electronic relaxation pathway in which the two positive charges (corresponding to the Auger/autoionization final state) are delocalized over two neighboring molecular entities..$^{25-27}$ The usually reactive and short-lived di-cationic pairs can be identified by a high-kinetic energy shoulder in the respective Auger-electron spectra. ${ }^{22,28}$ Core-level-induced ICD in aqueous solutions is an approximately 5\% effect compared to the local Auger process; however, local and non-local processes become equally important in the presence of ionization-induced proton dynamics. The latter is true for strong hydrogen bonding as in $\mathrm{H}_{2} \mathrm{O}(\mathrm{aq})^{21}$ and $\mathrm{H}_{2} \mathrm{O}_{2}(\mathrm{aq}){ }^{29}$ whereas proton dynamics is less important for weaker hydrogen-bonded $\mathrm{NH}_{3}(\mathrm{aq})$ and glycine ${ }^{30}$ in basic aqueous solution.

Several technical developments have paralleled liquid-jet PE spectroscopy, most noticeably ambient-pressure (AP) PE spectroscopy, ${ }^{31-35}$ i.e., PE spectroscopy at pressures above the vapor pressure of most common solvents (typically $>20$ mbar), as well as the detection of photoelectrons through a thin (typically few graphene layers) membrane. ${ }^{36-38}$ These techniques, unlike the liquid-jet technique, enable PE spectroscopy studies from the solid-solution interface and thus allow for in operando studies of (photo)electrochemical processes occurring at electrodes in solution. As depicted in Fig. 1(B), AP-PE spectroscopy is applied to a thin water film deposited on top of the solid surface, ${ }^{31}$ often an electrode material such as TM-oxide. Two methods have been used for preparing liquid films of only few-layer thickness, i.e., smaller than the electron mean free path, even for electron energies near $100 \mathrm{eV}$ kinetic energy. One approach is the so-called dip and pull method to create a stable nanometers-thick aqueous electrolyte film on a working-electrode surface..$^{32,35}$ The other method is the condensation of a thin electrolyte film on a solid substrate, stabilized at suitable relative humidity and temperature. ${ }^{31,34,39}$ In the case of a photoelectron-transparent graphene membrane, sustaining pressure differences of six orders of magnitude, ${ }^{36,38}$ the membrane can simultaneously serve as a support for a thin film of the electrode material. Both types of films will be measured with $\mathrm{SOL}^{3}$.
Our motivation for establishing $\mathrm{SOL}^{3}$ for photoemission studies from liquid phase at BESSY II was driven by the following quests:

(1) maximization of detection sensitivity in order to measure signal from poorly soluble molecules;

(2) simultaneous detection of electrons emitted at different angles from the solution-air/vacuum interface;

(3) detection of electrons emitted from the solid-solution interface;

(4) detection of all photoemission channels, i.e., valence and core-level photoionization, as well as local and non-local autoionization channels upon corehole ionization and excitation, from the solution-solid interface;

(5) measurements at AP conditions.

Valence and core-level PE spectra have been reported for many atomic ions and for a large number of most relevant organic and inorganic molecules which are sufficiently soluble in water. ${ }^{2,4}$ Typically, concentrations $>0.1-0.5 \mathrm{M}$ were needed in previous experiments; in the case of surface-active molecules, much lower concentrations are sufficient. ${ }^{40} \mathrm{We}$ were unable to obtain adequate signal-to-noise valence PE spectra from many molecular systems of biological and catalytic interest, including nucleobases, porphyrins, and hemin. Also, no electron signal from any TM-oxide nanoparticle (NP) dissolved in water has been detected with our previous LiquidPES setup. Below, we show that with $\mathrm{SOL}^{3}$ high-quality (resonant) PE spectra and the derived partial electron-yield $\mathrm{X}$-ray absorption (PEY-XA) spectrum from 6-nm diameter $\mathrm{TiO}_{2}$ NPs (5 wt. \% in water) can be obtained, using the identical X-ray photon flux and focal size as in previous experiments with the old LiquidPES setup, within an appreciably short collection time. Previously, core-level PE spectra from TM-oxide NPs have been reported for aqueous-phase $\mathrm{TiO}_{2}(20 \mathrm{~nm}$ diameter $)^{41}$ and $\mathrm{SnO}_{2}(20 \mathrm{~nm})^{42}$ using also state-of-the-art electron detection. The first photoelectron spectra from NPs (aq) were for $\mathrm{SiO}_{2}(10 \mathrm{~nm})^{43}$ which can be prepared at rather high concentration, and the signal is appreciably large. Photoelectron angular distributions from aqueous solution provide insight into the effect on hydrogen-bonding on the orbital structure and in addition reveal information on electron scattering in solution. ${ }^{20}$ Specifically, the broadening of the PAD, and hence decrease of the anisotropy parameter $\beta$, with respect to the gas-phase distribution is directly connected with the electron elastic-to-inelastic scattering cross-sectional ratio. For sufficiently small electron kinetic energies, $<100 \mathrm{eV}$, for which a significant anisotropy in the PAD remains, information about the orbital characters can be extracted from the PE spectra. Reduction in the anisotropy with respect to the gas phase can be used to estimate the electron inelastic mean free path (IMFP) relative to the elastic mean free path (EMFP). Furthermore, if the kinetic energy is within the range of small inelastic mean free path, interfacial molecular orientation can be revealed. To date, however, aqueous-phase PADs and their implications have been little explored. The one case for which $\beta$ 's have been determined based on the measurement of full PADs is the oxygen 1s ionization of neat liquid water, using a liquid microjet. ${ }^{20}$ In fact, the experimentally determined 
$\beta$ 's have provided insight into electron scattering, enabling an estimate of the related probing depth into water. Specifically, the related electron attenuation length (EAL) was found to not exhibit the minimum near $100 \mathrm{eV}$ kinetic energy of the universal mean free path curve; values for $<100 \mathrm{eV}$ stay low, at least down to approximately $20 \mathrm{eV}$ kinetic energy. ${ }^{19,20}$ The electron analyzer used with $\mathrm{SOL}^{3}$ can be operated in a so-called angular mode which enables the simultaneous detection of photoelectrons within $\pm 11^{\circ}$ angle. Due to the overall high electron transmission data-acquisition time for a full oxygen $1 \mathrm{~s}$, water PAD (covering angles $0^{\circ}-90^{\circ}$ ) will be reduced by a factor of approximately 20 as compared to earlier measurements. ${ }^{20}$

In our previous photoemission studies from liquid microjets, we have demonstrated that the complete understanding of the electronic structure of aqueous solutions requires the measurement of both photoelectron and autoionization spectra. These very powerful spectroscopic methods, including aforementioned non-local ICD, will also be extremely useful to apply to the solution-solid interface. The challenge here is to detect Auger electrons at their given kinetic energies $(\sim 500 \mathrm{eV}$ for $\mathrm{O} 1 \mathrm{~s}$ core-hole relaxation, $\sim 400 \mathrm{eV}$ for $\mathrm{N} 1 \mathrm{~s},{ }^{30}$ and $\sim 700 \mathrm{eV}$ for $\mathrm{Fe} 2 \mathrm{p}^{23}$ ) which cannot be altered, and hence the electron probing depth into the solution is fixed, and it is rather small. ${ }^{19,20}$ To date, these second-order electrons, from which the PEY-XA spectra can be readily generated, have not been spectroscopically detected from any solid-solution interface. Such spectra have been instead approximated by measuring the electron current at an anode electrode within the electrolyte solution; the particular experiment was performed for neat water at a gold surface. ${ }^{44,45}$ Lacking the spectroscopic information though, electrons originating from different processes, and being emitted at a different distance from the electrode, cannot be distinguished. An exploration of the spectroscopic routes is a major goal once $\mathrm{SOL}^{3}$ is upgraded and equipped for preparing and characterizing thin water and electrolyte films on suitable substrates. Likewise, for the study of (photo)electrochemical reactions at the electrode-solution interface, particularly water splitting, a suitable flow cell will be implemented.

The following section is a technical description of $\mathrm{SOL}^{3}$, presently equipped for conducting liquid-jet soft X-ray photoemission studies. First measurements with the new setup, exemplified for neat water and $\mathrm{TiO}_{2} \mathrm{NP}$ aqueous solution, are presented, highlighting the efficient electron detection using $\mathrm{SOL}^{3}$.

\section{SOL ${ }^{3}$-DESCRIPTION AND SPECIFICATIONS}

$\mathrm{SOL}^{3}$ consists of three main components, the interaction chamber (IC), the electron analyzer (EA), and a differential pumping unit (DP), all shown in Fig. 2. The setup can be easily moved between different beamlines at BESSY II.

\section{Interaction chamber}

The $27 \times 27 \times 42 \mathrm{~cm}^{3}$ of non-magnetic stainless steel houses the liquid jet mounted on an $x-y-z$ manipulator, holds the hemispherical electron analyzer, and provides a port for the X-ray beam entering the chamber. Additional ports accommodate cooling traps, observation windows, ion gauges, and simple diagnostics to facilitate positioning of the X-ray beam. Spare ports are available for future installation of crystal holders, gas inlets, (photo)electrochemical cell, for adaption of a load-lock chamber, and for feeding in UV/optical light. The chamber is evacuated down to $10^{-6}$ mbar within $20 \mathrm{~min}$ by a $1600 \mathrm{l} / \mathrm{s}$ non-corrosive turbomolecular pump; this pump is mounted on a separate $300 \mathrm{~mm}$ diameter stainless-steel cylinder connected to the interaction chamber. When running the liquid jet (typically 10-25 $\mu \mathrm{m}$ diameter) experiment, the pressure in the IC is as low as $10^{-4} \mathrm{mbar}$, using one or more cold traps (metal cylinders with approximately $1000 \mathrm{~cm}^{2}$ surface area each) filled with liquid nitrogen. Studies from liquid jets in a higher vapor-pressure environment, or any liquid-surface (stabilized at the respective relative humidity) ambient-pressure studies, can be performed up to pressures equivalent to 20 mbar $\mathrm{H}_{2} \mathrm{O}$ or 50 mbar $\mathrm{N}_{2}$ at the sample position. ${ }^{35,46}$

Emitted electrons can be detected at any angle between $90^{\circ}$ and $0^{\circ}$ (one quadrant), including the magic angle 54. $7^{\circ},{ }^{47}$ with respect to the polarization vector of the synchrotron light which is typically in the floor plane. For that $\mathrm{SOL}^{3}$ is equipped with an easy-to-use swing mechanism, allowing for quick rotation of the EA about the synchrotron-light beam (see Fig. 2). Rotation is done manually, using a pulley. To rotate the analyzer, breaking the vacuum is necessary.

The IC is mounted on an adjustable aluminum frame, designed to achieve maximal mobility and flexibility in terms of experimental needs. This enables to operate $\mathrm{SOL}^{3}$ at different beamlines to match the given experimental requirements. The whole setup is on wheels, allowing for easy relocation. Once the setup is connected to the beamline port and anchored on four solid pillars, the $\mu \mathrm{m}$-fine-positioning can be carried out. Rails (in $\mathrm{x}$ - and y-direction) and a swivel joint are mounted for translation in $\mathrm{x}$ - and $\mathrm{y}$ directions and for rotating the whole

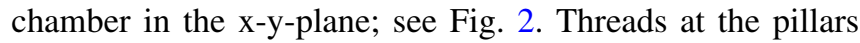
allow for height adjustment ( $\mathrm{z}$ direction) and for rotating in the $\mathrm{x}-\mathrm{z}$ - and $\mathrm{y}-\mathrm{z}-\mathrm{plane}$.

Unwanted magnetic fields, in particular, the earth magnetic field, are compensated by a Helmholtz-coil arrangement. Each of the three pairs of coils is powered separately. Currents are optimized to yield zero magnetic field, measured with a Hall sensor in front of the electron analyzer. The dimensions of the coils are as follows: pair 1 (with magnetic field along the beamline axis) has $54 \mathrm{~cm}$ diameter and $27 \mathrm{~cm}$ distance from the point where electrons are detected; pair 2 (along the analyzer axis) has $24 \mathrm{~cm}$ diameter at $27 \mathrm{~cm}$ distance; and coil pair 3 (along jet axis) has $21 \mathrm{~cm}$ diameter at $27 \mathrm{~cm}$ distance.

\section{Electron analyzer}

The electron spectrometer Scienta Omicron R4000 HiPP-2 is a high-resolution hemispherical electron energy analyzer developed for photoelectron spectroscopy using excitation energies ranging from $\mathrm{UV}$ to hard $\mathrm{X}$-ray, suited for operation under ambient-pressure conditions up to tens of millibars. Detailed specifications can be 


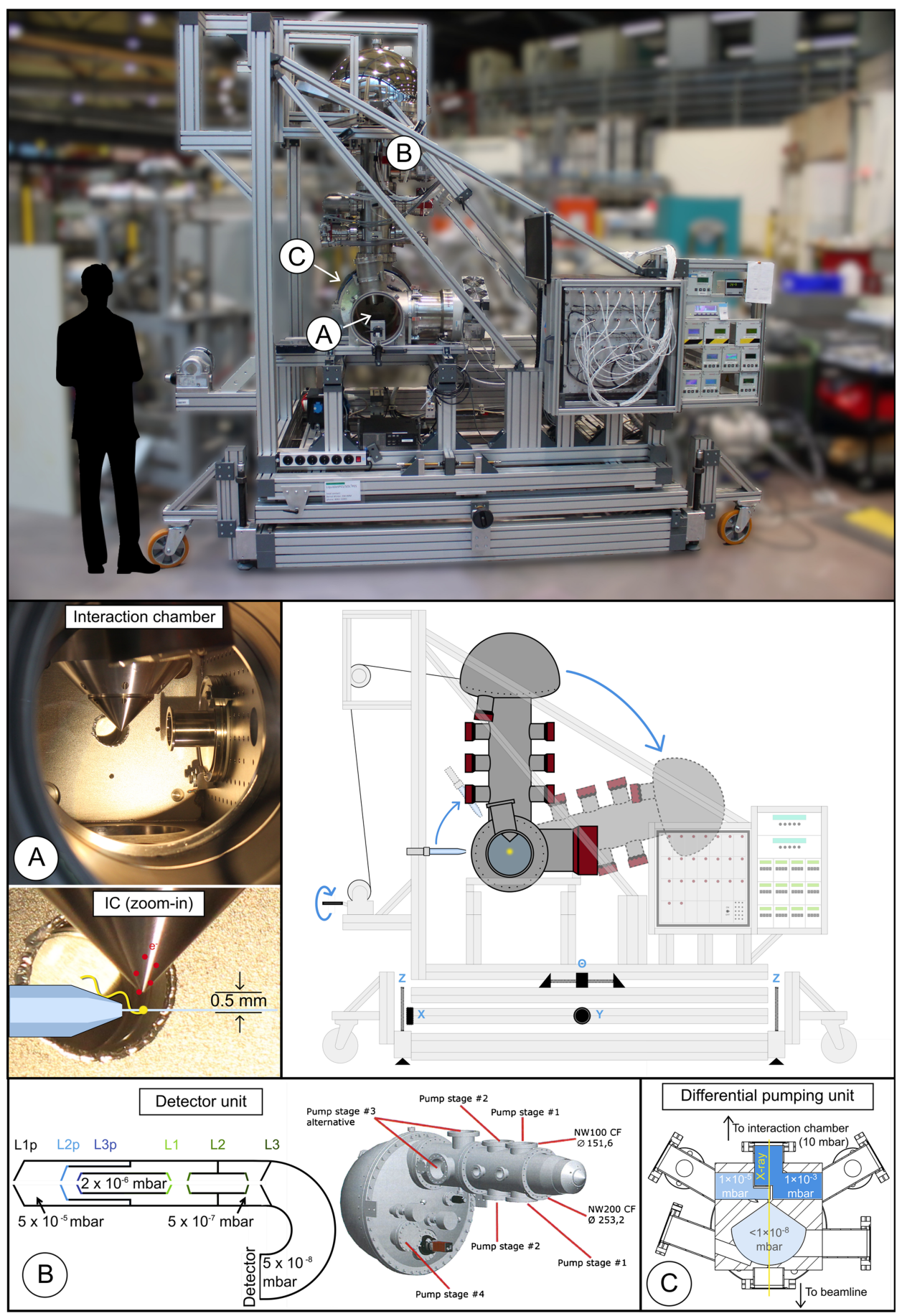

FIG. 2. $\mathrm{SOL}^{3}$ setup with the hemispherical electron analyzer in two different detection positions $\left(90^{\circ}\right.$ and $15^{\circ}$ emission angles). Components for (fine-) adjusting of the frame and the analyzer are indicated. (A) Interaction chamber. (B) Sketch of the analyzer and electron lens (e.g., L1) and pre-lens (e.g., L1p) systems. Typical pressures are shown. (C) Top view of the threecomponent differential pumping unit. This unit is mounted on the backside of the main vacuum chamber and cannot be seen in the main figure.

found in Ref. 46 and on the Scienta Omicron website: http://www.scientaomicron.com/en/products/354/1179 (date: June 2017). Our spectrometer consists of a pre-lens and an electrostatic lens system for imaging electrons of different energies (5-6000 eV) which are then guided into the section containing the two electrostatic hemispheres (radius of the outer hemisphere $200 \mathrm{~mm}$ ). Electrons with a given preset pass energy $( \pm 8 \%$ distribution) are imaged onto a $2 \mathrm{D}$ detector, consisting of a micro-channel plate and a CCD camera. AP-operation of the R4000 HIPP-2 analyzer is accomplished with the help of multi-stage pumping. Below, the most important features and specifications of the analyzer are described.

\section{Lens modes}

The Scienta R4000 HiPP-2 offers two data acquisition modes: transmission mode and angular mode. The transmission mode is the standard method of lens operation. It produces an image of the sample at the detector and is intended for large-spot analysis of relatively homogeneous samples. The transmission mode maximizes the total transmission through the lens at a constant magnification of about 5. The spatial resolution in transmission mode is approximately $300 \mu \mathrm{m}$. The angular mode is a special way of lens operation in which the emission angle distribution is imaged. The novel refocusing design of the pre-lens enables the simultaneous detection over 
$22^{\circ}$. The angular resolution is dependent on the emission spot size, with smaller spot sizes giving the highest resolution. At $0.1 \mathrm{~mm}$ spot size, the angular resolution is better than $0.3^{\circ}$.

\section{Energy range and resolution}

The total energy detection range is $5-6000 \mathrm{eV}$ in transmission mode and 10-6000 eV in angular mode. Nine different sizes of the entrance slit to the hemisphere are available: 0.2 $\times 25 \mathrm{~mm}^{2}$ (curved), $0.3 \times 30 \mathrm{~mm}^{2}$ (straight), $0.3 \times 25 \mathrm{~mm}^{2}$ (curved), $0.5 \times 30 \mathrm{~mm}^{2}$ (straight), $0.5 \times 25 \mathrm{~mm}^{2}$ (curved), 0.8 $\times 25 \mathrm{~mm}^{2}$ (curved), $1.5 \times 30 \mathrm{~mm}^{2}$ (straight), $2.5 \times 30 \mathrm{~mm}^{2}$ (straight), and $4.0 \times 30 \mathrm{~mm}^{2}$ (straight). The best energy resolution is $15 \mathrm{meV}$ at $500 \mathrm{eV}$ kinetic energy, using a $10 \mu \mathrm{m}$ beamline slit, and $70 \mathrm{meV}$ at $10000 \mathrm{eV}$ kinetic energy.

\section{Ambient-pressure operation}

Three aperture cones, of 300, 500, and $800 \mu \mathrm{m}$ pinhole diameter, can be mounted in front of the analyzer entrance (the section housing the electrostatic lenses), serving as differential pumping stage. The larger angle acceptance in the case of larger diameters allows for higher electron transmission but leads to higher pressure in the region of the first imaging lens system, ruling out measurements under AP conditions. The entire analyzer unit comprises four separately pumped sections, three of which are part of the lens system, and the fourth is the hemisphere chamber. Each lens section is equipped with two $250 \mathrm{l} / \mathrm{s}$ turbo pumps, and a single turbo pump is mounted at the analyzer chamber; turbo pumps are backed up by $10 \mathrm{l} / \mathrm{s}$ scroll pumps.

\section{Detector unit}

The final detector producing electron signals consists of two multichannel plates (MCPs) and a $40 \mathrm{~mm}$-diameter phosphorous screen which generates a 2D (energy by position/angle) image of the electrons. This image is recorded by a CCD camera which is mounted on a viewport on the non-vacuum side. The detector image registered by the CCD camera corresponds to a rectangle of 560 simultaneous energy channels and 460 channels in the spatial/angular dispersion and is controlled by the choice of lens mode.

\section{Differential pumping unit}

A $(15 \mathrm{~cm})^{3}$ cubic three-compartment chamber connects the IC with the refocusing chamber (RC) of the synchrotron beamline; see Fig. 2(C). Both connections are via flexible bellows. The compact design of the DP allows for use of $\mathrm{SOL}^{3}$ at beamlines with different focal lengths; minimum length between the exit flange of the last beamline chamber and the point of light interaction with the sample is $65 \mathrm{~cm}$. The purpose of the DP is to maintain a $<10^{-8}$ mbar pressure in the $\mathrm{RC}$ for any pressure in the IC. In the extreme case of ambientpressure conditions, this is accomplished with the help of three $250 \mathrm{l} / \mathrm{s}$ turbo pumps, one for each compartment, and in addition the compartment closest to the IC is equipped with a liquid nitrogen cold trap. Small apertures aligned along the light path connect the compartments; pinhole sizes are 1, 2, and $3 \mathrm{~mm}$ (from IC to RC). To further assist in guiding the light beam through the DP, small areas around the pinhole are covered with a film that fluoresces when hit by the X-ray beam.

\section{PHOTOEMISSION MEASUREMENTS}

This section presents two liquid-jet PE measurements obtained with $\mathrm{SOL}^{3}$ during a dedicated 2-weeks commissioning period in 2016. Photoelectron spectra from both neat liquid water (with $0.05 \mathrm{M} \mathrm{NaCl}$ added to increase conductivity) and aqueous 6-nm $\mathrm{TiO}_{2}$ NPs (anatase phase, 5 wt. \%) were measured by introducing a $24-\mu \mathrm{m}$ liquid jet into the IC. Experiments were conducted at the U49/2-PGM-1 undulator beamline of BESSY II, Berlin. This beamline provides a reasonably small focal size of $20 \times 80 \mu \mathrm{m}^{2}$ at high photon flux/intensity $\left(5 \cdot 10^{13} \mathrm{ph} / \mathrm{s} / 0.1 \mathrm{~A} / 0.1 \%\right.$ bandwidth at $150 \mathrm{eV}$ photon energy) and assures that the (water) gas-phase signal contribution to the total (photo)electron signal is much smaller than the signal from aqueous phase. The jet velocity was approximately $60 \mathrm{~m} \mathrm{~s}^{-1}$, and the jet temperature was $12{ }^{\circ} \mathrm{C}$, similar to our previous studies. ${ }^{3}$ Electrons were detected with the R4000 HIPP-2 electron analyzer, where we used a 500- $\mu \mathrm{m}$ diameter orifice (entrance to the EA) at approximately $500 \mu \mathrm{m}$ distance from the liquid jet. These distances and apertures are found to be a good balance between the electron collection efficiency, determined by the solid angle, and the pumping efficiency in the lens region. While the electron-collection efficiency increases with aperture size and decreases with aperture distance, the pumping efficiency increases with aperture distance and decreases with aperture size. ${ }^{48,49}$ A pressure of $10^{-7}$ mbar was obtained in the lens region, and $10^{-8}$ mbar in the MCP region, while the pressure in the IC was $5 \cdot 10^{-4}$ mbar. ${ }^{50}$ The detection angle was set to $90^{\circ}$ with respect to the light polarization vector. Further details are described below for each experiment.

We first present in Fig. 3 the valence PE spectrum of neat liquid water, exhibiting signals from all three outer-valence

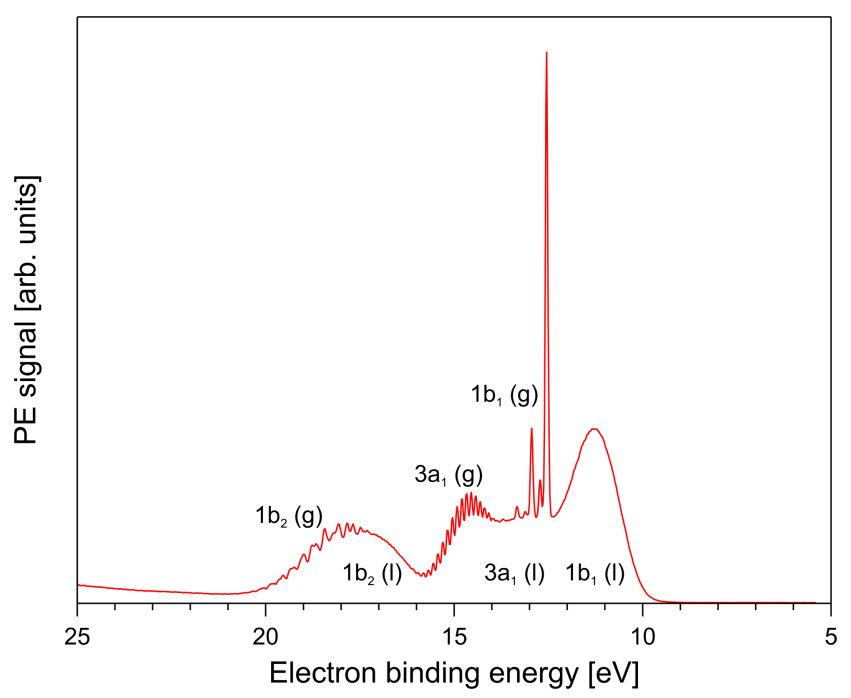

FIG. 3. Valence photoelectron spectrum of neat liquid water measured at $165 \mathrm{eV}$ photon energy. Emission contributions from the three valence orbitals, $1 b_{1}, 3 a_{1}$, and $1 b_{2}$, are labeled. Subscripts $l$ and $g$ indicate the contributions from liquid- and gas-phase ionization, respectively. Binding energies are with respect to vacuum. 
orbitals: $1 b_{1}(11.31 \mathrm{eV}$ binding energy $(\mathrm{BE})), 3 \mathrm{a}_{1}(\sim 13.5 \mathrm{eV}$ $\mathrm{BE})$, and $1 \mathrm{~b}_{2}(17.4 \mathrm{eV} \mathrm{BE}) .^{51}$ The spectrum has been collected within only $200 \mathrm{~s}$. The photon energy was $165 \mathrm{eV}$ (in the $300 \mathrm{~mA}$ BESSY II top-up mode), and the energy resolution of the beamline was $10 \mathrm{meV}$. The detector slit of the electron analyzer was set to $0.5 \times 30 \mathrm{~mm}^{2}$, corresponding to $25 \mathrm{meV}$ nominal energy resolution for $20 \mathrm{eV}$ pass energy used here. Under the given experimental conditions, the signal contribution due to ionization of gas-phase water to the total signal is approximately $10 \%$ which is best seen when considering the ionization of the water $1 b_{1}$ orbitals (see labels in the figure); these two contributions have only little spectral overlap. In all our previous measurements, ${ }^{4}$ it was impossible to obtain a smooth line spectrum within reasonably short collection time. Not only were collection times long, on the order of $1 \mathrm{~h}$, but also the energy resolution of both X-rays and the electron analyzer had to be fairly small in order to obtain sufficiently large photon flux and electron transmission, respectively. To be more specific, the count rates in Fig. 3 are approximately a factor of 30 larger than in previous measurements from a water liquid jet, for a comparable liquid-to-gas signal ratio and similar experimental settings. And at the same time, the large transmission of the EA, even at the chosen moderate energy resolution of $25 \mathrm{meV}$ for $20 \mathrm{eV}$ pass energy, is sufficient to well resolve the vibrational states of gas-phase water; these are the distinct peaks between 12 and $13 \mathrm{eV} \mathrm{BE}$ for water $1 b_{1}$, and the comb-like structures on the high-BE sides of the water $3 \mathrm{a}_{1}$ and $1 b_{2}$ orbitals. Although the water spectrum in Fig. 3 does not provide any new information on the electronic structure of liquid water, the spectrum gives an idea on how the increased detection sensitivity can be exploited for future photoemission studies from low-concentration (aqueous) solutions. We also anticipate that low electron signals from the solid-solution interface can be detected with fair statistics in cases where the electron inelastic mean free path is small. To illustrate the new possibilities offered by $\mathrm{SOL}^{3}$, we next present photoemission spectra from $\mathrm{TiO}_{2}$ NPs in aqueous solution.

The main challenge in performing photoemission studies from aqueous-phase metal-oxide NPs is to identify a spectral signature of the NP-solution interface. Titanium dioxide NPs are of particular importance because of the central role this material plays for artificial photosynthesis, capable to perform catalytic water splitting. A yet debated issue is the exact nature of the immediate interface, and there is accumulating evidence that both dissociated and molecular water exist in the first monolayer. One picture that arises is that the energetic cost of water dissociation is reduced via the formation of some hydrogen-bonded hydroxyl-water complex, ${ }^{31,52}$ $\mathrm{HO} \cdot \mathrm{H}_{2} \mathrm{O}$. These strongly bound complexes act as nucleation centers for water adsorption. Experimentally, one thus anticipates observing at least three different types of signal in the $\mathrm{O}$ 1s photoemission spectra, from NP lattice oxygen, from the $\mathrm{HO} \cdots \mathrm{H}_{2} \mathrm{O}$ interfacial complex, and from bulk water, both in direct and resonant photoemission (RPE). No solute-derived $\mathrm{TiO}_{2}$ NP signal from on- and off-resonant measurements has been detected in our earlier attempts.

Figure 4(A) shows a series of photoemission spectra from a $5 \mathrm{wt}$ \% $\%$ aqueous solution of $6-\mathrm{nm} \mathrm{TiO}_{2}$ anatase NPs measured across the onset of the oxygen 1 s resonance. Total collection time was only $30 \mathrm{~min}$. NPs were stabilized by adding nitric acid (0.1 M). The bottom spectrum, measured at $529 \mathrm{eV}$ photon energy, which is just slightly below any $\mathrm{O} 1 \mathrm{~s}$ resonance, is essentially the valence spectrum of neat water shown in Fig. 3. That is, contributions from solute ionization are very small, almost unnoticeable. A strong peak at $\sim 520 \mathrm{eV} \mathrm{KE}$ is due to ionization of the water $\mathrm{O} 1 \mathrm{~s}$ orbital with second-order light $(1058 \mathrm{eV})$ which was deliberately used here for an unequivocal energy calibration. When increasing the photon energy to $530.5 \mathrm{eV}$, the appearance of new small spectral features in the 504-511 eV KE range can be noticed [highlighted in red (1), green (2), and light-blue (3)]. Near $533.5 \mathrm{eV}$ photon energy, a strong signal increase indicates the onset of the X-ray absorption pre-peak of bulk water with maximum at $535 \mathrm{eV} .^{53}$ This energy corresponds to the water $\mathrm{O} 1 \mathrm{~s} \rightarrow 4 \mathrm{a}_{1}$ excitation, and the observed electron signal is due to the subsequent Auger decay. ${ }^{54}$

We have demonstrated in several previous studies on TM atomic ions (aq) that electron-signal enhancements in the valence photoelectron spectra at resonant $2 p$ core-level excitation can identify otherwise undetectably small signals. ${ }^{23,24}$ This is also the case here, reflected in aforementioned peaks 1, 2, and 3. Peak 3 can be assigned to a spectator Auger decay of the stabilizing $\mathrm{NO}_{3}{ }^{-}$(aq) anions; there is also a contribution from $\mathrm{NO}_{3}{ }^{-}$spectator Auger electrons at $515 \mathrm{eV}$ kinetic energy. The assignment to $\mathrm{NO}_{3}{ }^{-}$photoemission is based on comparative measurements at the oxygen 1s edge from $\mathrm{HNO}_{3}$ aqueous solutions, presented in Fig. 4(B) for $0.5 \mathrm{M}$ concentration. Below we provide evidence that $\mathrm{NO}_{3}{ }^{-}$in bulk aqueous solution, and $\mathrm{NO}_{3}{ }^{-}$adsorbed at the NPs have slightly different electronic structures. Peak 2 in Fig. 4(A) is due to Augerelectron emission from the (lattice) oxygen coordinated to the titanium atoms, in agreement with a previous assignment from experimental oxygen 1s absorption spectra from crystalline $\mathrm{TiO}_{2}$ in contact with water; see, e.g., Ref. 55. One notices that peak 2 moves to larger kinetic energies with decreasing photon energy, appearing at similar binding energies as peak $\mathbf{3}$ once the excitation photon energy is below $531.5 \mathrm{eV}$. This suggests that peak $\mathbf{2}$ is not due to a single species, and it cannot be the signature of a given species in an identical chemical environment. The weak peak 1 can be assigned to an interfacial species, resulting from the interaction of water molecules with the $\mathrm{TiO}_{2}$-NP surface, as will be detailed with the help of the O 1s PEY-XA spectra.

PEY-XA spectra from the $5 \mathrm{wt} \% \mathrm{TiO}_{2} \mathrm{NP}$ and the $0.5 \mathrm{M}$ $\mathrm{HNO}_{3}$ aqueous solutions are presented at the right side of panels (A) and (B) of Fig. 4. These spectra were obtained by integration of the signal intensities of each RPE spectrum, in the 529-534 eV photon-energy region. The occurrence of two maxima in the PEY-XA spectrum from the NP aqueous solution at 530.8 (peak A) and 532.2 eV (peak B) photon energies, which are absent for neat liquid water (spectra not shown here; see, e.g., Ref. 53), is unequivocally assigned to the photoemission from solute. These signals correlate with peaks 1-3 in the RPE spectra. Peak $\mathbf{A}$ is hence primarily due to lattice oxygen, peak $\mathbf{2}$, and peak $\mathbf{B}$ largely arises from adsorbed $\mathrm{NO}_{3}{ }^{-}$, peak $\mathbf{3}$, and from adsorbed $\mathrm{OH}^{-}$, peak $\mathbf{1}$. Our assignment to adsorbed $\mathrm{NO}_{3}{ }^{-}$is based on the fact that at the low concentrations of the present study, all $\mathrm{HNO}_{3}$ molecules will dissociate into 

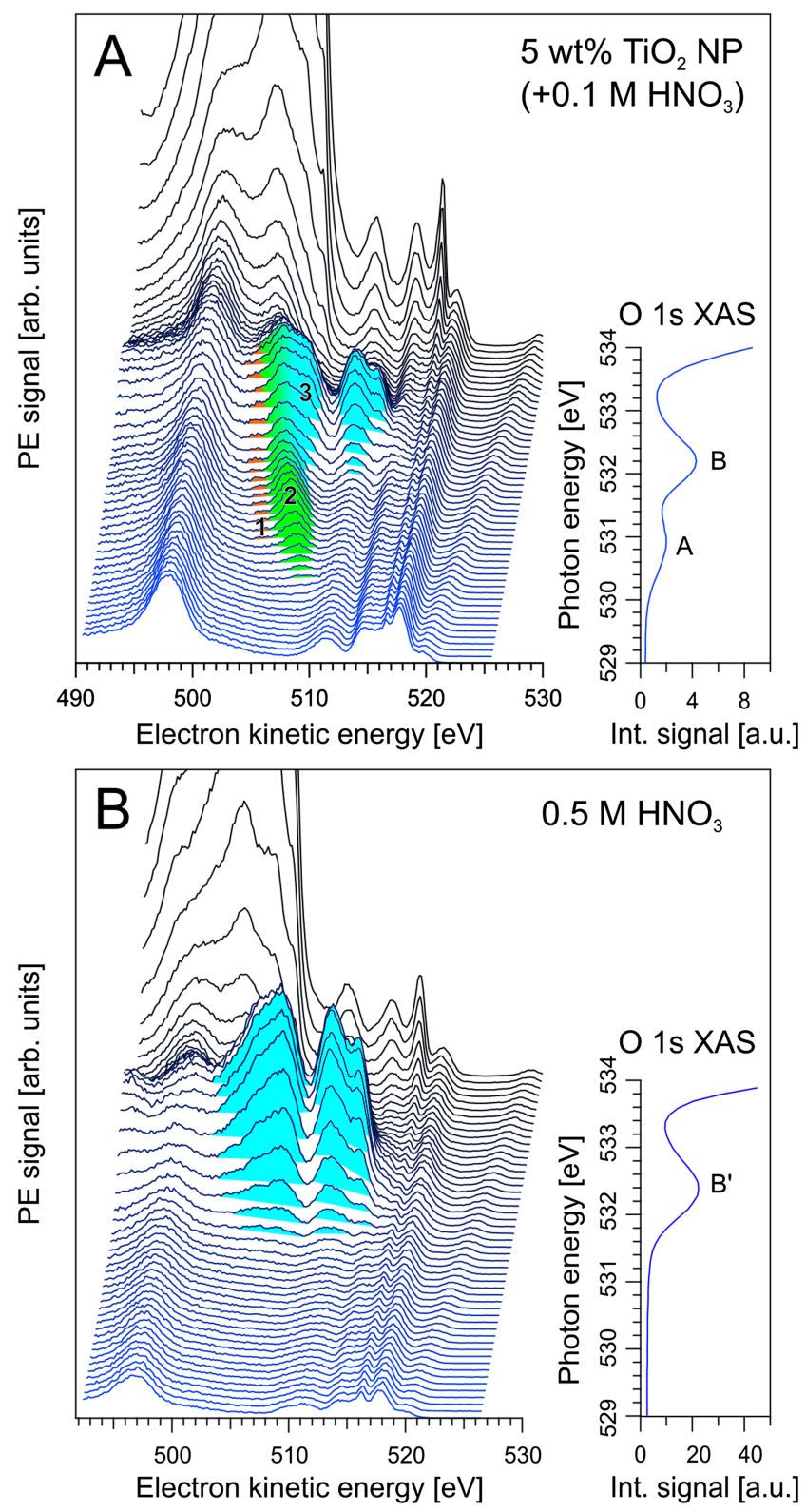

FIG. 4. (A) Oxygen 1s resonant photoelectron spectra from 6-nm diameter $\mathrm{TiO}_{2} \mathrm{NP}$ (anatase phase) in aqueous solution. The fraction of the partial electron yield X-ray absorption (PEY-XA) spectrum obtained by integration of the intensities of the respective PE spectra is shown at the right side. The contribution from the $\mathrm{TiO}_{2}$ NPs is highlighted in red and green, and the contribution from the $\mathrm{HNO}_{3}$ stabilizer is marked in blue. (B) Oxygen 1s RPE spectra and derived PEY-XA spectrum from $0.5 \mathrm{M} \mathrm{HNO}_{3}$ aqueous solution.

$\mathrm{NO}_{3}{ }^{-}$(aq) and $\mathrm{H}^{+}(\mathrm{aq})\left[\mathrm{H}_{3} \mathrm{O}^{+}(\mathrm{aq})\right] \cdot{ }^{17}$ A large fraction of the nitrate anions will inevitably interact with the positively charged NP surface, for which we have measured a positive zeta potential. A more direct experimental distinction between absorbed and fully hydrated $\mathrm{NO}_{3}{ }^{-}$is an observed 150-meV energy difference of peak B [Fig. 4(A)] and the corresponding peak $\mathbf{B}^{\prime}$ [Fig. 4(B)]; the larger energy is measured for the fully hydrated $\mathrm{NO}_{3}{ }^{-}$. The qualitative explanation for adsorbed $\mathrm{OH}^{-}$is the low $\mathrm{pH}$, such that free $\mathrm{OH}^{-}$molecules do not exist in solution. Also, our O 1s RPE spectra from $\mathrm{NaOH}$ aqueous solution (see, e.g., Ref. 54) exhibit $\mathrm{OH}^{-}$signal in the very similar energy range as observed for the NP solutions.
Our preliminary measurements from TM-oxide NPs in aqueous solution clearly demonstrate the great value of RPE spectroscopy for future studies on the behavior of water adsorption on metal oxides for an advancement of engineering molecular systems in energy and materials research.

In summary, $\mathrm{SOL}^{3}$ is a well suited tool to address many of the current scientific and technological challenges which are based on processes in aqueous phase. For that an understanding of the various aspects of electronic-structure interactions is required, exploiting the unique versatility, design, and parameters of $\mathrm{SOL}^{3}$.

\section{ACKNOWLEDGMENTS}

The authors thank Olaf Schief (W\&P GEAT GmbH, Berlin) for designing and constructing the frame of $\mathrm{SOL}^{3}$. We are also thankful to Detlef Müller and his team from the workshop at the Freie Universität Berlin and to Florian Gores from HZB for assistance in designing the differential pumping section. The authors also thank the workshop at BESSY II, and particularly Oliver Schappeit, for assistance during the commissioning phase, and we also acknowledge support from the BESSY II staff for assisting in the operation of the beamline. Scienta Omicron engineers are acknowledged for support during implementation of this instrumentation. B.W. and R.S. gratefully acknowledge support from the Deutsche Forschungsgemeinschaft (DFG) (Collaborative Research Centre 1109), and B.W. and M.N.P. also thank the DFG Research Unit FOR 1789 for financial support. R.S. acknowledges the DFG for awarding an Emmy-Noether Grant (No. SE 2253/3-1). H.A. thanks the Ain Shams University for a Ph.D. grant. E.F.A. acknowledges the financial support from HZB and the European Research Council Grant No. 279344.

${ }^{1}$ B. Winter and M. Faubel, Chem. Rev. 106, 1176 (2006).

${ }^{2}$ B. Winter, Nucl. Instrum. Methods Phys. Res., Sect. A 601, 139 (2009).

${ }^{3}$ R. Seidel, S. Thürmer, and B. Winter, J. Phys. Chem. Lett. 2, 633 (2011).

${ }^{4}$ R. Seidel, B. Winter, and S. E. Bradforth, Annu. Rev. Phys. Chem. 67, 283 (2016).

${ }^{5}$ M. Faubel, B. Steiner, and J. P. Toennies, J. Electron Spectrosc. Relat. Phenom. 95, 159 (1998).

${ }^{6}$ K. R. Siefermann, Y. X. Liu, E. Lugovoy, O. Link, M. Faubel, U. Buck, B. Winter, and B. Abel, Nat. Chem. 2, 274 (2010).

${ }^{7}$ Y. Tang, H. Shen, K. Sekiguchi, N. Kurahashi, T. Mizuno, Y. I. Suzuki, and T. Suzuki, Phys. Chem. Chem. Phys. 12, 3653 (2010).

${ }^{8}$ A. T. Shreve, T. A. Yen, and D. M. Neumark, Chem. Phys. Lett. 493, 216 (2010).

${ }^{9}$ F. Buchner, A. Lübcke, N. Heine, and T. Schultz, Rev. Sci. Instrum. 81, 113107 (2010).

${ }^{10}$ A. Lübcke, F. Buchner, N. Heine, I. V. Hertel, and T. Schultz, Phys. Chem. Chem. Phys. 12, 14629 (2010).

${ }^{11}$ R. Seidel, S. Thürmer, J. Moens, P. Geerlings, J. Blumberger, and B. J. Winter, J. Phys. Chem. B 115, 11671 (2011).

${ }^{12}$ D. Ghosh, A. Roy, R. Seidel, B. Winter, S. Bradforth, and A. I. J. Krylov, J. Phys. Chem. B 116, 7269 (2012).

${ }^{13}$ D. Nolting, N. Ottosson, M. Faubel, I. V. Hertel, and B. Winter, J. Am. Chem. Soc. 130, 8150 (2008).

${ }^{14}$ T. Lewis, B. Winter, A. C. Stern, M. D. Baer, C. J. Mundy, D. J. Tobias, and J. C. Hemminger, J. Phys. Chem. C 115, 21183 (2011).

${ }^{15}$ T. Lewis, M. Faubel, B. Winter, and J. C. Hemminger, Angew. Chem., Int. Ed. 50, 10178 (2011). 
${ }^{16}$ N. Ottosson, M. Faubel, S. E. Bradforth, P. Jungwirth, and B. J. Winter, J. Electron Spectrosc. Relat. Phenom. 177, 60 (2010).

${ }^{17}$ T. Lewis, B. Winter, A. C. Stern, M. D. Baer, C. J. Mundy, D. J. Tobias, and J. C. Hemminger, J. Phys. Chem. B 115, 9445 (2011).

${ }^{18}$ S. Ghosal, J. C. Hemminger, H. Bluhm, B. S. Mun, E. L. D. Hebenstreit, G. Ketteler, D. F. Ogletree, F. G. Requejo, and M. Salmeron, Science 307, 563 (2005).

${ }^{19}$ Y.-I. Suzuki, K. Nishizawa, N. Kurahashi, and T. Suzuki, Phys. Rev. E 90, 010302 (2014)

${ }^{20}$ S. Thürmer, R. Seidel, M. Faubel, W. Eberhardt, J. C. Hemminger, S. E. Bradforth, and B. Winter, Phys. Rev. Lett. 111, 173005 (2013).

${ }^{21}$ P. Slavíček, B. Winter, L. S. Cederbaum, and N. V. Kryzhevoi, J. Am. Chem. Soc. 136, 18170 (2014).

${ }^{22}$ P. Slavíček, N. Kryzhevoi, E. F. Aziz, and B. Winter, J. Phys. Chem. Lett. 7, 234 (2016).

${ }^{23}$ S. Thürmer, R. Seidel, W. Eberhardt, S. E. Bradforth, and B. Winter, J. Am. Chem. Soc. 133, 12528 (2011).

${ }^{24}$ R. Seidel, K. Atak, S. Thürmer, E. F. Aziz, and B. Winter, J. Phys. Chem. B 119, 10607 (2015).

${ }^{25}$ U. Hergenhahn, J. Electron Spectrosc. Relat. Phenom. 184, 78 (2011).

${ }^{26}$ L. S. Cederbaum, J. Zobeley, and F. Tarantelli, Phys. Rev. Lett. 79, 4778 (1997).

${ }^{27}$ T. Jahnke, J. Phys. B: At., Mol. Opt. Phys. 48, 082001 (2015).

${ }^{28}$ S. Thürmer, M. Ončák, N. Ottosson, R. Seidel, U. Hergenhahn, S. E. Bradforth, P. Slavíček, and B. Winter, Nat. Chem. 5, 590 (2013).

${ }^{29}$ I. Unger, S. Thürmer, D. Hollas, E. F. Aziz, B. Winter, and P. J. Slavíček, J. Phys. Chem. C 118, 29142 (2014).

${ }^{30}$ I. Unger, D. Hollas, R. Seidel, S. Thürmer, E. F. Aziz, P. Slavíček, and B. Winter, J. Phys. Chem. B 119, 10750 (2015).

${ }^{31}$ G. Ketteler, S. Yamamoto, H. Bluhm, K. Andersson, D. E. Starr, D. F. Ogletree, H. Ogasawara, A. Nilsson, and M. Salmeron, J. Phys. Chem. C 111, 8278 (2007).

${ }^{32}$ E. J. Crumlin, H. Bluhm, and Z. Liu, J. Electron Spectrosc. Relat. Phenom. 190, 84 (2013).

${ }^{33}$ O. Karslioglu, S. Nemsak, I. Zegkinoglou, A. Shavorskiy, M. Hartl, F. Salmassi, E. M. Gullikson, M. L. Ng, C. Rameshan, B. Rude, D. Bianculli, A. A. Cordones, S. Axnanda, E. J. Crumlin, P. N. Ross, C. M. Schneider, Z. Hussain, Z. Liu, C. S. Fadley, and H. Bluhm, Faraday Discuss. 180, 35 (2015).

${ }^{34}$ C. H. Wu, R. S. Weatherup, and M. B. Salmeron, Phys. Chem. Chem. Phys. 17, 30229 (2015).

${ }^{35}$ S. Axnanda, E. J. Crumlin, B. Mao, S. Rani, R. Chang, P. G. Karlsson, M. O. M. Edwards, M. Lundqvist, R. Moberg, P. Ross, Z. Hussain, and Z. Liu, Sci. Rep. 5, 9788 (2015).
${ }^{36}$ A. Kolmakov, D. A. Dikin, L. J. Cote, J. Huang, M. K. Abyaneh, M. Amati, L. Gregoratti, S. Guenther, and M. Kiskinova, Nat. Nanotechnol. 6, 651 (2011).

${ }^{37}$ J. Kraus, R. Reichelt, S. Guenther, L. Gregoratti, M. Amati, M. Kiskinova, A. Yulaev, I. Vlassiouk, and A. Kolmakov, Nanoscale 6, 14394 (2014).

${ }^{38}$ J. J. Velasco-Velez, V. Pfeifer, M. Hävecker, R. S. Weatherup, R. Arrigo, C.-H. Chuang, E. Stotz, G. Weinberg, M. Salmeron, R. Schlögl, and A. Knop-Gericke, Angew. Chem., Int. Ed. 54, 14554 (2015).

${ }^{39}$ M. Salmeron and R. Schlogl, Surf. Sci. Rep. 63, 169 (2008).

${ }^{40}$ P. R. Tentscher, R. Seidel, B. Winter, J. J. Guerard, and J. S. Arey, J. Phys. Chem. B 119, 238 (2015).

${ }^{41}$ M. J. Makowski, R. P. Galhenage, J. Langford, and J. C. Hemminger, J. Phys. Chem. Lett. 7, 1732 (2016).

${ }^{42}$ I. Jordan, A. B. Redondo, M. A. Brown, D. Fodor, M. Staniuk, A. Kleibert, H. J. Worner, J. B. Giorgi, and J. A. van Bokhoven, Chem. Commun. 50, 4242 (2014)

${ }^{43}$ M. A. Brown, R. Seidel, S. Thurmer, M. Faubel, J. C. Hemminger, J. A. van Bokhoven, B. Winter, and M. Sterrer, Phys. Chem. Chem. Phys. 13, 12720 (2011).

${ }^{44}$ J.-J. Velasco-Velez, C. H. Wu, T. A. Pascal, L. F. Wan, J. Guo, D. Prendergast, and M. Salmeron, Science 346, 831 (2014).

${ }^{45}$ D. Schon, J. Xiao, R. Golnak, M. F. Tesch, B. Winter, J. J. Velasco-Velez, and E. F. Aziz, J. Phys. Chem. Lett. 8, 2087 (2017).

${ }^{46}$ S. K. Eriksson, M. Hahlin, J. M. Kahk, I. J. Villar-Garcia, M. J. Webb, H. Grennberg, R. Yakimova, H. Rensmo, K. Edstrom, A. Hagfeldt, H. Siegbahn, M. O. M. Edwards, P. G. Karlsson, K. Backlund, J. Ahlund, and D. J. Payne, Rev. Sci. Instrum. 85075119 (2014).

${ }^{47}$ S. Hüfner, Photoelectron Spectroscopy: Principles and Applications (Springer-Verlag, Berlin, Heidelberg, New York, London, Paris, Tokyo, Hong Kong, Barcelona, Budapest, 1995).

${ }^{48}$ D. F. Ogletree, H. Bluhm, G. Lebedev, C. S. Fadley, Z. Hussain, and M. Salmeron, Rev. Sci. Instrum. 73, 3872 (2002).

${ }^{49}$ H. Bluhm, J. Electron Spectrosc. Relat. Phenom. 177, 71 (2010).

${ }^{50}$ J. T. Newberg, J. Åhlund, C. Arble, C. Goodwin, Y. Khalifa, and A. Broderick, Rev. Sci. Instrum. 86085113 (2015).

${ }^{51}$ B. Winter, R. Weber, W. Widdra, M. Dittmar, M. Faubel, and I. V. Hertel, J. Phys. Chem. A 108, 2625 (2004).

${ }^{52}$ C. E. Patrick and F. Giustino, Phys. Rev. Applied 2, 014001 (2014); eprint arXiv:1607.07992v1.

${ }^{53} \mathrm{Ph}$. Wernet, D. Nordlund, U. Bergmann, M. Cavalleri, M. Odelius, H. Ogasawara, L. A. Naslund, T. K. Hirsch, L. Ojamae, P. Glatzel, L. G. M. Pettersson, and A. Nilsson, Science 304, 995 (2004).

${ }^{54}$ E. F. Aziz, N. Ottosson, M. Faubel, I. V. Hertel, and B. Winter, Nature 455, 89 (2008).

${ }^{55}$ G. Vanderlaan, Phys. Rev. B 41, 12366 (1990). 